\title{
Multilayer Dielectric Elastomer Actuators with Ion Implanted Electrodes
}

\author{
A. Punning*, S. Akbari, M. Niklaus, and H. Shea \\ EPFL, LMTS, rue Jaquet Droz 1, 2002 Neuchatel, Switzerland
}

\begin{abstract}
We present the design, fabrication process and characterization of multilayer miniaturized polydimethylsiloxane (PDMS)-based dielectric elastomer diaphragm actuators. The conductive stretchable electrodes are obtained by lowenergy metal ion implantation. To increase force, decrease the required voltage, and avoid dielectric breakdown, we present here a technique to fabricate multilayer devices with embedded electrodes with complex shapes. By implanting electrodes on a partially cured PDMS film, then casting on it the next layer of PDMS, it is possible to have the compliant electrodes "molded" inside PDMS. Using custom shadow masks allows defining electrodes of any shape or size, we report sizes down to $0.1 \mathrm{~mm}$. The minimal distance between independent electrodes inside the PDMS is limited solely by the breakdown voltage of PDMS and can be also as small as $0.1 \mathrm{~mm}$. Using this approach, we have fabricated miniature compact devices consisting of several independent dielectric elastomer actuators on a single PDMS film. Applying different voltages to the separate actuators allows to achieve complicated movements of the whole device, e.g. to act as a 3-DOF parallel manipulator. A distinctive feature of the multi-layer actuators is that they attain similar strain with lower voltage than the single-layer actuators of the same thickness. We report on a $3 \mathrm{~mm}$ diameter 2 -axis beam steering device combining three actuators.
\end{abstract}

Keywords: Dielectric elastomer actuator, ion implantation, PDMS

\section{INTRODUCTION}

\subsection{Dielectric elastomers}

Dielectric elastomer electroactive polymer (DEAP) actuators consist of an elastic dielectric layer sandwiched between two electrodes. The electrodes must be stretchable and conform to the size and shape of the dielectric membrane. Applying a voltage between the electrodes creates an electrostatic pressure. This pressure compresses the dielectric layer. If the bulk modulus of the dielectric is high (its volume does not change), its area increases. Details about the DEAP actuators are described in ref [1].

It is difficult to find any applications of such a self-expansible sheet without a supporting structure. In order to use it as an actuator, it is generally incorporated into a mechanical structure with pre-strain or an initial force applied to the membrane. In its electrically actuated state the membrane expands and allows the structure to deform due to the initial force that is no longer countered; in electrically inactive state it remains contracted. The force applied or released by the membrane can then be translated to movement of other structures. Depending on how the boundaries of the membrane are attached, the structure can contract, bend, buckle, etc. It is also possible to have an initial state with no pre-strain or no applied force, and the expansion of the film leads to a buckling deformation.

One possible configuration of a DEAP actuator is the so-called diaphragm or buckling actuator. The elastomer film is stretched on a frame and bulges out of the plane. In order to define the direction of bulging, a pre-deformation or force is applied to the membrane already in its (electrically) inactive state. Then, a voltage applied between the electrodes induces surface expansion and promotes the actual buckling of the membrane. This effect has been exploited in the socalled diaphragm actuators, by stretching elastomer films on frames and applying different pressures of air between its sides, or placing under it a hemispheric support, see e.g. refs [1,2].

*andres.punning@ut.ee; +372 7374832

Electroactive Polymer Actuators and Devices (EAPAD) 2011, edited by Yoseph Bar-Cohen, Federico Carpi, Proc. of SPIE Vol. 7976, 79760U · C 2011 SPIE · CCC code: 0277-786X/11/\$18 · doi: 10.1117/12.880484 


\subsection{Ion implantation}

Ion implantation is a material engineering process by which the ions of one material are accelerated in an electric field and bombarded into another, target material. This process is commonly used to change the physical, chemical, or electrical properties of the target ${ }^{3,4}$. This technology can be easily exploited to fabricate highly compliant electrodes in the surface region of soft polymer elastomers. Target polymer is placed directly into the accelerating electric field. The plasma cloud is formed close to the target and the accelerated ions are bombarded into it. The heavy ions of metal penetrate into the polymer up to tens of nanometers and form conductive layer consisting of crystalline nanoparticles ${ }^{5-8}$. This technology is called Plasma Ion Immersion Implantation (PIII) ${ }^{9}$. As the ions can be manipulated magnetically, it is possible to filter out the macroparticles.

Earlier we have developed a novel method to form highly compliant electrodes in/on polymers by implanting metal ions at low energies $(1$ to $5 \mathrm{keV}){ }^{5-8}$. This technology has been used to fabricate millimeter-size vertical stroke membrane micro-actuators whose efficiency is the similar to the macro-scale actuators. They are made of 20-50 $\mu \mathrm{m}$ thick PDMS film with working voltage of a few kilovolts. In order to obtain the conductive electrodes to the PDMS films, we used a custom-made Filtered Cathode Vacuum Arc (FCVA) system. It is based on RHK Technology ARC 20 pulsed arc source. It operates in pulse mode and its beam size is $1 \mathrm{~cm}^{2}$. The system consists of a conductive cathode where plasma is created, a high-voltage trigger to initiate the arc, an anode to extract the plasma towards the filter and a solenoid operating as a macroparticle filter. The process of ion implantation operates in a vacuum chamber at the pressure below $10^{-5}$ mbar. The working principle of this FCVA system and the characterization of the compliant electrodes produced with it are thoroughly described in refs [5-8].

A distinctive feature of ion implantation is the possibility to fabricate compliant electrodes of filigree pattern. This is achieved by covering the target partially with a shadow mask, made of metal or fabricated photolitographically directly on the target. The electrodes are formed solely at the areas exposed to plasma. The accuracy and the minimal linear dimensions of electrodes are limited by the technology of fabrication of the mask. (Actually, the experience shows that the minimal linear dimensions should not be less than thickness of the mask.) We report on as narrow as $100 \mu \mathrm{m}$ linear dimensions of the compliant electrodes ${ }^{10}$, and $100 \mu \mathrm{m}$ distances between separate electrodes using steel masks fabricated by laser cutting technology.

This approach enables fabrication of miniature compact devices consisting of several independent dielectric elastomer actuators on a single PDMS film. In order to demonstrate its capabilities and shortages we fabricated miniature devices for steering a mirror, driven with three DEAP actuators. One expected field of usage of the device is Optical Coherence Tomography (OCT). Specifications of interest for the OCT system are the size of the device (a few $\mathrm{mm}$ ); and speed of scanning (up to $100 \mathrm{~Hz}$ ).

\section{DESIGN CONSIDERATIONS}

It is possible to design myriads of different configurations of miniature PDMS-based DEAP devices. The challenges to their fabrication are similar. Some of them, in addition to the technology of fabrication of compliant electrodes, are:

- The operating voltage of DEAP actuators depends on the thickness and relative dielectric permittivity of the dielectric, but noticeable actuation is achieved usually with voltage in the range of kilovolts. The breakdown voltage of the particular material or surrounding environment limits the minimal distance between separate actuating elements. Therefore it is efficient to form the independent electrodes inside of a material of higher dielectric strength.

- PDMS sticks easily to almost everything smooth, but forms permanent bonds only to limited materials, e.g. after special processing in oxygen plasma to itself and glass. Another relevant design consideration is the method of incorporation of the PDMS film to the supporting mechanical structure.

- The compliant electrodes obtained by ion implantation are more brittle than virgin PDMS, attaching them to the external wires is a delicate task, though we have found effective solutions.

- By definition, a DEAP actuator is a capacitor. When the distance between separate actuators is narrow, there emerges an additional unavoidable capacitance between electrodes simply because of their proximity to each other. Therefore the design of the high voltage power electronics controlling the device should be also considered. 
The design of our steering device is deducted from a parallel mechanism (also called a parallel robot) - a closed-loop kinematic chain mechanism consisting of a fixed base platform, connected to an end effector platform by means of a number of independent joints ${ }^{11}$. One possible approach is based on a thin mirror bonded to three vertical stroke membrane micro-actuators. Each actuator can independently move "up and down", thus orienting the mirror to the necessary angle. A much more challenging parallel mechanism consists of a single pre-deformed buckling actuator with a mirror attached to its top. The circular electrodes of the buckle are divided into several separate independent sectorshaped electrodes. This configuration acts as usual bulging actuator when all sectors are actuated identically. If the separate sectors are actuated differently, the 3-dimensional shape of the buckle will vary and its tangent plane at the point of attached mirror will incline as depicted in Figure. 1. Controlling at least 3 independent sectors of the bulge makes it possible to achieve complicated movements of the whole device and to control the angle of the mirror in its 2dimensional state space.

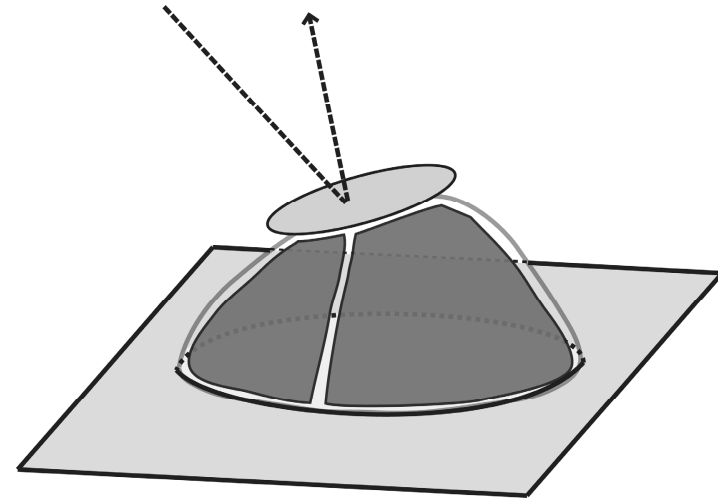

A

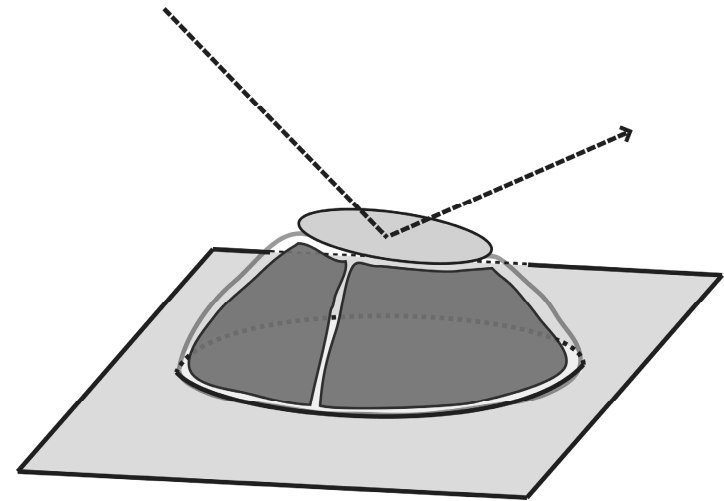

B

Figure.1. Changing the surface areas of separate sectors deforms the buckle and steers the mirror.

Earlier we have developed the full methodology of fabrication and characterization of buckling actuators of a 2-3 mm in diameter ${ }^{5,6}$. In order to utilize all previous fixtures and tools, the sizes of our device was chosen also to be 2-3 mm. For maximum performance, the inactive space between the separate electrodes was chosen to be as narrow as possible close to the limit of the technology of fabrication of the steel shadow masks $-100-150 \mu \mathrm{m}$.

It is self-evident that due to the low moisture-dependent dielectric strength of air it is impossible to use this device in air conditions, as the breakdown resulting with a spark will destroy the device at once. A possible way to overcome this shortage, is to conceal the separate electrodes inside PDMS. If they are inside PDMS film exactly at its half thickness, the resulting multi-layer device looks like two similar actuators bonded on top of another. This laminate attains similar strain with twice lower voltage than a single-layer actuator of the same (double) thickness. All electrodes on the outer surfaces of the device are connected to the common ground of all voltage sources. Here a few new important design considerations arise:

- For on-site testing the conductivity of the electrodes buried into PDMS, each of them should have at least two exposed contacts.

- It is possible to bond PDMS to itself, but not to the gold electrodes. Developing the process of concealing the electrodes into PDMS happened to be the most laborious part of this project.

A critical design consideration in the development of PDMS-based devices fabricated through multi-layer soft lithography is the method of bonding used to combine the molded layers. After polymerization and cross-linking, the hydrophobic surface of PDMS will not establish chemical bonds before its surface chemistry is altered. A number of PDMS bonding techniques have been reported in the literature over the last several years: oxidation with oxygen plasma, partial curing, uncured PDMS adhesive, corona discharge, etc. ${ }^{12}$. One of them - partial curing of PDMS - has shown to be an effective, inexpensive method for creating multi-layer devices ${ }^{13}$. The PDMS mix with the polymerization agent is cured at either a short period of time (30-60 min) or at a lower temperature (less than $60^{\circ} \mathrm{C}$ ). The PDMS layers become hardened enough to handle and remove from the molds, but remain un-crosslinked enough at the interfaces to form 
effective bonds with other partially cured details. Even though partial curing is limited for bonding fully cured layers, this simple and cost-effective method is well suitable for bonding the layers in our device.

Bonding a mirror in the center of the bulge is accomplished with magnets. Two tiny neodymium iron boron (NdFeB) disc magnets are placed exactly to the center of the bulging actuator. As the opposite poles of the magnets draw to each other, they compress PDMS slightly and will be firmly attached to it. The mirror is attached to the upper magnet with glue. Another alternative is to polish the magnet itself to be reflective. The third magnet placed at appropriate distance will generate the necessary pre-deformation of the bulge. There are two possible configurations of pre-deformation depending on the configuration of the magnets: the third magnet may repel other magnets and push the film up, or pull other magnets down, as depicted in Figure 2. The whole system operates following the principle of minimum energy described by Kofod et al. ${ }^{14}$ When actuators are energized (possibly different voltages on each), the bulge and magnets obtain the state of minimal potential energy.

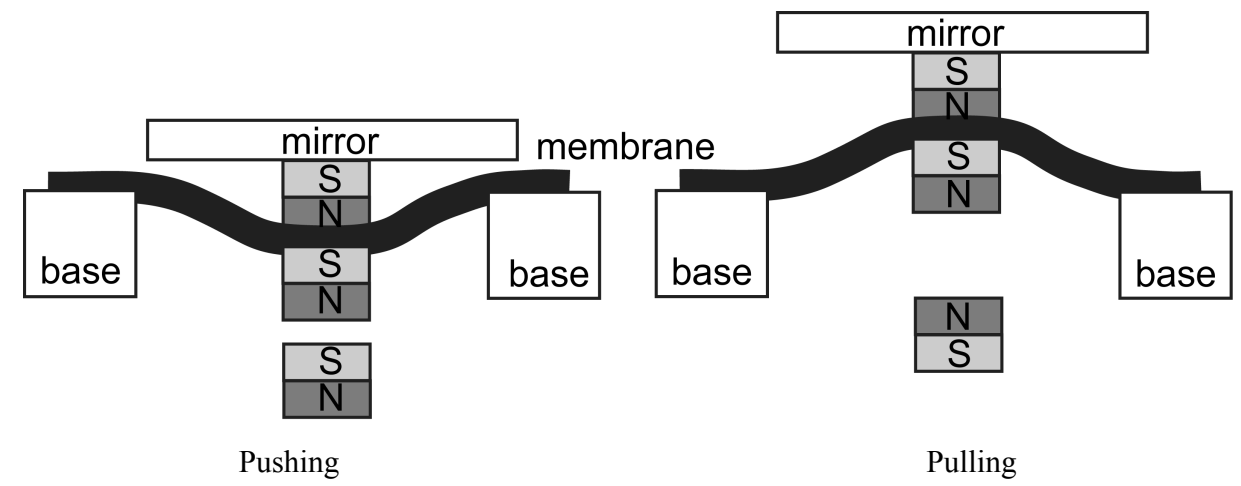

Figure 2. Two possible configurations of pre-deformation with magnets.

The thickness of the ion-implanted electrodes is a few tens of nanometers ${ }^{15}$. The most reliable method of attaching the external wires to the electrodes is to press a piece of foil to the contact areas. This should be done in gentle manner: if pressure is too weak, there will be no contact, if pressure is too strong, the PDMS film will deform too much and there will also be no contact. The contact areas of the electrodes concealed inside PDMS must be exposed during casting of PDMS layers.

\section{FABRICATION OF THE MULTILAYER DEVICE}

Fabrication of the multilayer device consists of several steps of casting PDMS layers and formation of compliant electrodes. It must be pointed out that timing and temperature regimes of this process are of essential importance. If the first PDMS layer is not cured enough, the process of ion implantation does not form a conductive electrode; if it is cured too much, the second layer will not bond to it. The parameters of the regimes - thicknesses of the casted PDMS layers, doses of the implantation, temperatures, etc. are pre-determined experimentally.

The steps of fabrication of the multilayer device are given in Figure 3.

1. PDMS Dow Corning Sylgard ${ }^{\circledR} 186$ is mixed with its polymerizing agent with mixing ratio 10:1. In order to diminish the viscosity of the resulting mixture, $90 \%$ of iso-octane (2-2-4-trimethylpenthane) is added. The admixture is thoroughly mixed and degassed 40 minutes in dessicator;

2. The first layer of PDMS is casted using the universal casting applicator Zehntner ZUA2000 on a thin polyimide film substrate. Iso-octane evaporates during polymerization; hence the thickness of the casted PDMS will decrease about 3 times. After polymerizing in oven at temperature $65^{\circ}$ for $25-30$ minutes the layer is hard enough for supporting the electrode, but still soft and half polymerized to form chemical bonds with the next layer of PDMS;

3. The electrodes on top of the first layer (electrode1) are created with ion-implantation technology using the appropriate shadow mask. It takes about 15 minutes to pump the vacuum chamber to the necessary level of pressure $\left(8 \cdot 10^{-7} \mathrm{mbar}\right)$; about 10 minutes for implantation of the desired number of pulses; and about 10 minutes to release the vacuum. The resulting layers are depicted in Figure 3A;

4. The contact areas of the electrodes are covered with small flakes of $25 \mu \mathrm{m}$ thick PTFE as depicted in Figure 3B; 


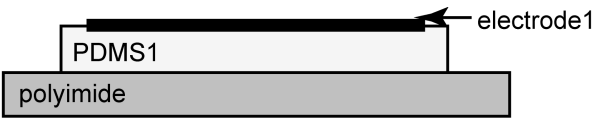

A
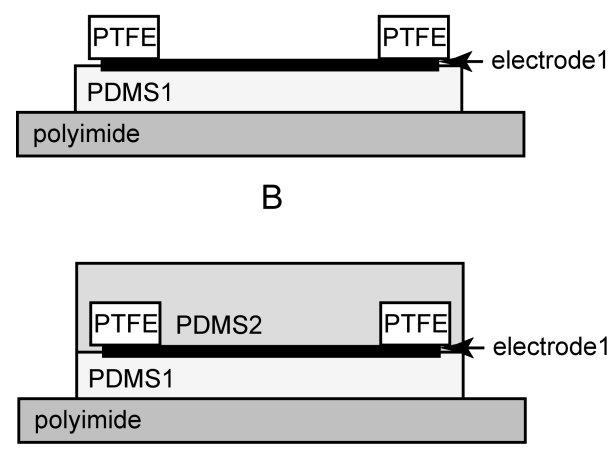

C

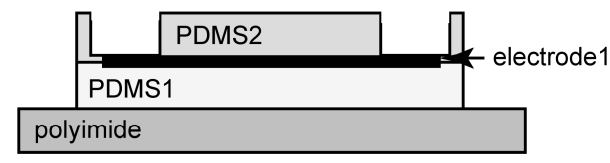

D

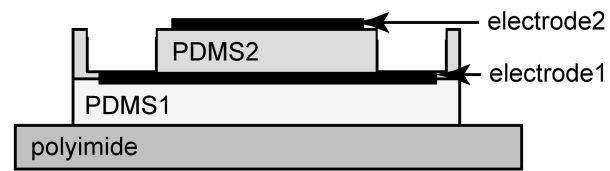

$\mathrm{E}$

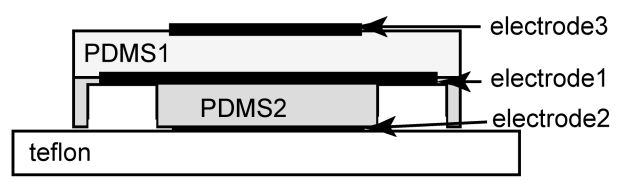

$\mathrm{F}$

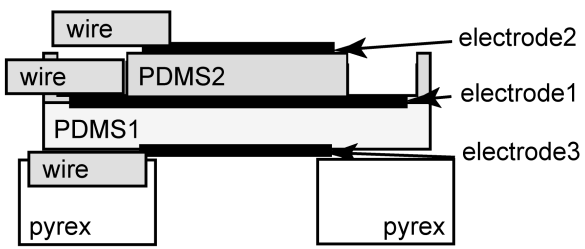

G

Figure 3. Fabrication of the 2-layer device.

5. The second layer of PDMS is casted over the first layer with the electrode and over the PTFE flakes, as depicted in Figure 3C;

6. The PTFE flakes are removed at once, exposing the contact areas of the implanted electrodes, as depicted in Figure 3D;

Up to this step the process is very time- and temperature-critical, otherwise the two layers will not bond together, or the conductive electrode will not form, or the thicknesses of the two layers of PDMS will not be equal. It must be taken into account that the process of polymerization of the first layer of PDMS continues, though slowly, during implantation of the electrode. The following steps are not so critical and can be completed later.

7. The resulting two-layer film is cured as recommended in the Sylgard 186 Product Information, e.g. 4 hours at temperature $65^{\circ}$. The result is a homogeneous PDMS film with conductive compliant electrode concealed in the middle;

8. The electrode on top of the second layer of PDMS (electrode2) is created using the ion-implantation technology and the appropriate shadow mask (Figure 3D);

9. In order to create the third compliant electrode, the PDMS film is removed from the polyimide substrate and overturned onto some nonstick surface, e.g. Teflon film (Figure 3E);

10. Finally, to fabricate a bulging device, the film is overturned again onto a glass or plastic substrate having a circular hole of appropriate diameter. Wires made of thin foil are attached to the exposed surfaces of the electrodes (Figure 3G).

Following the recipe given above, we have fabricated devices of different layouts consisting of 40 - $50 \mu \mathrm{m}$ PDMS film with compliant electrodes at the half of thickness and both outer surfaces. The thicknesses were measured with interferometric optical profiler Wyco 1100 produced by Veeco Instruments Inc. The accuracy of fabrication and deviation of thickness of the film was better than $\pm 2 \mu \mathrm{m}$. Two different layouts of the electrodes depicted in Figure 4 show, that the implanted electrodes are almost transparent, and the electrodes of the middle layer have two exposed contact areas in order to test their conductivity on-site. 


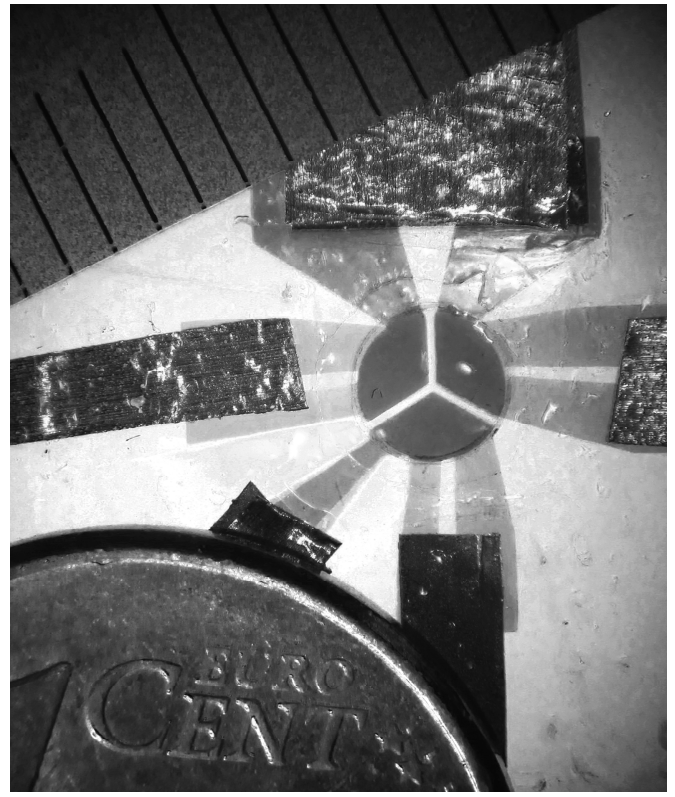

A

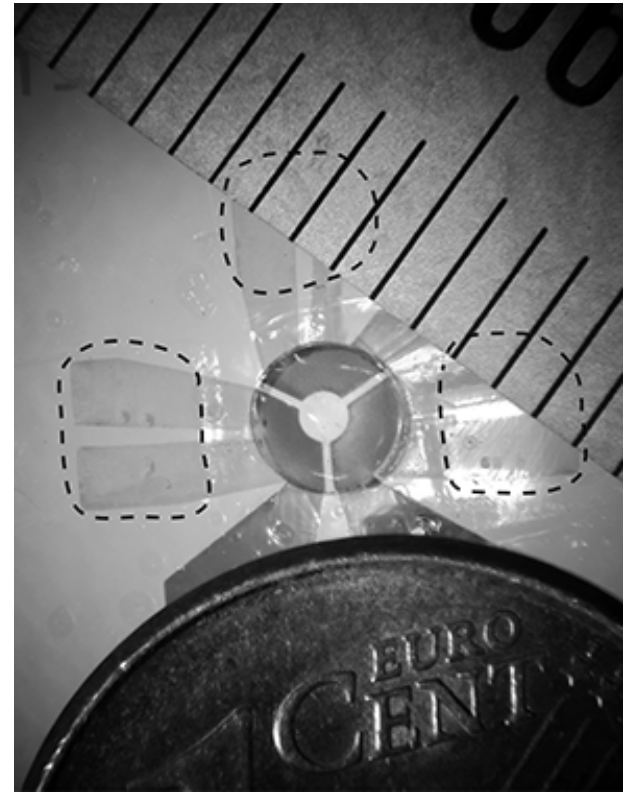

B

Figure 4. Two different layouts of 3 electrodes.

The scale of ruler is in millimeters, the diameter of 1 euro cent coin is $16.25 \mathrm{~mm}$.

A - with the wires made of foil; B - the exposed contact areas of the middle layer are marked with dashed line.

\section{CHARACTERIZATION}

We present the graphs of tilt angles of mirror with different voltage values. The 3 voltages were generated using 3 Stanford PS350 high voltage DC Power Supplies driven by NI Labview. This voltage source is precise, but slow, therefore does not allow measuring the frequency characteristics of the device. The angles of inclination of the device were measured using the PSM2-10 position sensing module and OT-301 precise position sensing amplifier manufactured by On-Track Photonics Inc. The three voltages applied to the three actuators were changed cyclically between zero and voltage value $\mathrm{U}:(\mathrm{U}, 0,0) \rightarrow(\mathrm{U}, \mathrm{U}, 0) \rightarrow(0, \mathrm{U}, 0) \rightarrow(0, \mathrm{U}, \mathrm{U}) \rightarrow$ etc. as depicted in Figure 5A. The inclination angles of the mirror with $\mathrm{U}$ values $700 \mathrm{~V}, 900 \mathrm{~V}$, and $1100 \mathrm{~V}$ are given in Figure $5 \mathrm{~B}$. This device is capable to incline the mirror up to \pm $3^{\circ}$ and shows good repeatability.

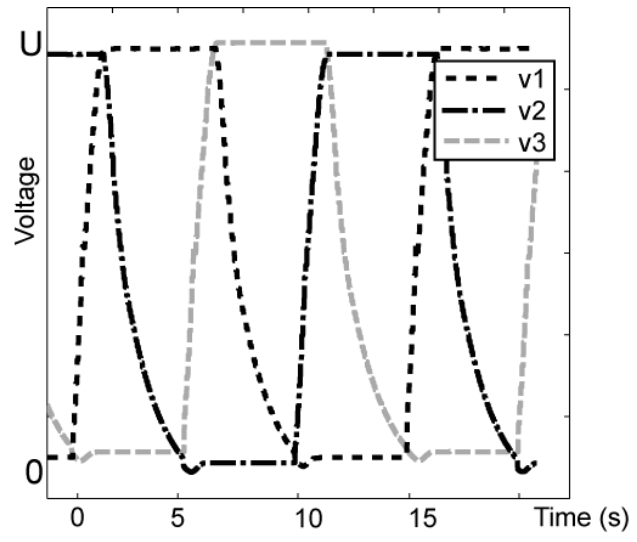

A

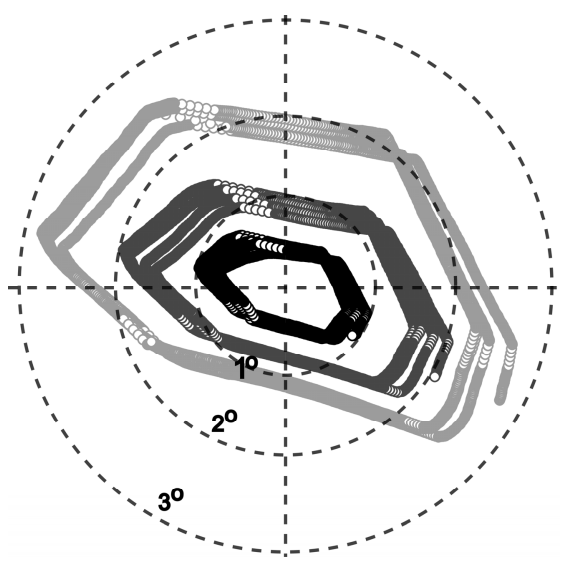

$\mathrm{B}$

Figure 5. A - voltages controlling three actuators; $\mathrm{B}$ - inclination angles of the mirror. 
Ideally, the shape of the graphs given in Figure 5B should be a regular hexagon. The discrepancy indicates that the performances of the actuators are not equal. Nevertheless, by controlling the voltages it is possible to achieve all angles in the state space of the device.

As described hereinabove, we have two opposite configurations of the device depending on the orientation of the magnet creating the pre-deformation of the membrane, see Figure 2. Two different devices, both in two different states of inclination are given in Figure 6. The diameter and thickness of the disc magnets holding the mirror are respectively 1 $\mathrm{mm}$ and $0.5 \mathrm{~mm}$. The diameter of the circle of the device depicted in Figure $6 \mathrm{~A}$ is $2 \mathrm{~mm}$; the third hidden magnet pulls the membrane down. In the case of the device given in Figure $6 \mathrm{~B}$ the diameter is $3 \mathrm{~mm}$ and the pre-deformation of the membrane is "up". The experiments show that if the membrane is convex, the range of achieved angles is wider, while if concave, the repeatability is better.
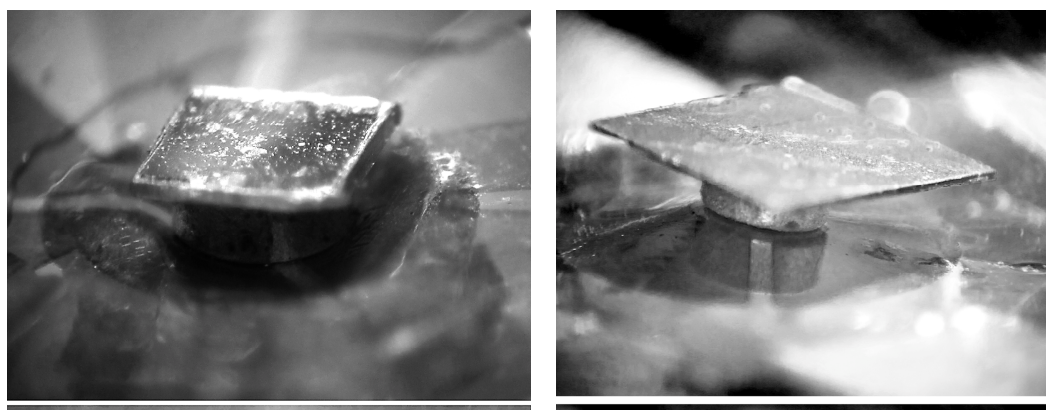

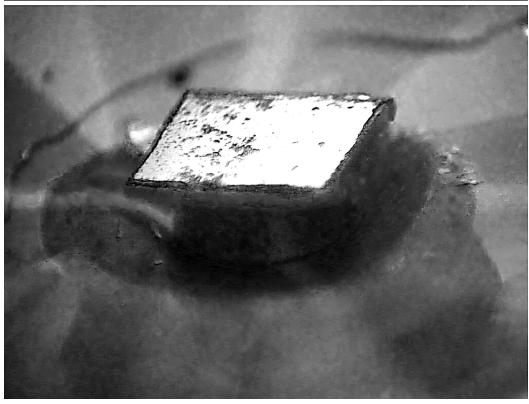

A

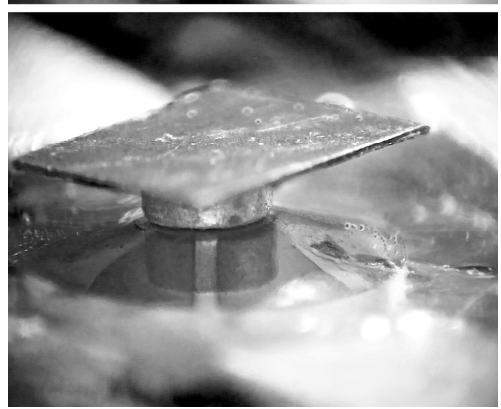

B

Figure 6. Two devices in different states of inclination.

A -device of $2 \mathrm{~mm}$ diameter bulging down; B - device of $3 \mathrm{~mm}$ diameter bulging up.

\section{CONCLUSIONS}

We have demonstrated that ion implantation makes it is possible to fabricate millimeter-scale compact DEAP devices consisting of a single 40-50 $\mu \mathrm{m}$ thick PDMS film with several electrodes of filigree shapes. Applying different voltages to the separate areas of the film allows achieving complicated movements of the whole device. In order to prevent destruction of the device by high voltage breakdown, the independent compliant electrodes are concealed at the half of thickness of the film. This is accomplished by molding the device layer-by-layer, while processing the electrodes.

The advantages of multilayer approach are:

- It makes it possible to form independent DEAP actuators with high working voltage very close to each other. Even though theoretically the interval between actuators is defined by breakdown voltage of PDMS (about $1 \mathrm{kV}$ per $10 \mu \mathrm{m}$ ), from practical viewpoint it is limited by the technology of fabricating the shadow masks.

- The double-layer actuator attains similar strain with twice lower voltage than a single-layer actuator of the same (double) thickness, consequently the driving voltage can be lower;

- If the voltage driving the device is lower, the gap between the separate independent electrodes can be even more narrow

- As the high voltage electrodes are concealed inside PDMS, and the electrodes exposed to outwards are all connected to the common ground of the voltage sources, the device can be considered safe by means of high voltage. 


\section{ACKNOWLEDGEMENTS}

This work was supported by the Indo Swiss Joint Research Programme (ISJRP), grant \#122948.

\section{REFERENCES}

[1] Carpi, F., De Rossi, D., Kornbluh, R., Pelrine, R. E., Sommer-Larsen, P., [Dielectric Elastomers as Electromechanical Transducers], Elsevier Science, (2008).

[2] Pelrine, R., Sommer-Larsen, P., Kornbluh, R., Heydt, R., Kofod, G., Pei, Q., Gravesen, P., “Applications of dielectric elastomer actuators." [Smart Structures and Materials: Electroactive Polymer Actuators and Devices], ed. BarCohen, Y., Proc. SPIE , 4329, 335-349, (2001).

[3] Cuomo, J. J., Haber, S. M., Kaufman, H. R., [Handbook of Ion Beam Processing Technology: Principles, Deposition, Film Modification and Synthesis], William Andrew Publishing, (1989).

[4] Kondyurin, A., Bilek, M., Ion Beam Treatment of Polymers, Elsevier Science, (2008).

[5] Dubois, P., Rosset, S., Koster, S., Stauffer, J., Mikhailov, S., Dadras, M., de Rooij, N.-F., Shea, H., "Microactuators based on ion implanted dielectric electroactive polymer (EAP) membranes," Sensors and Actuators A: Physical, vol. 130-131, pp. 147-154 (2006).

[6] Rosset, S., Niklaus, M., Dubois, P., Shea, H., "Large-Stroke Dielectric Elastomer Actuators With Ion- Implanted Electrodes", Journal of Microelectromechanical Systems, vol. 18, num. 6, 1300-1308 (2009).

[7] Rosset, S., Niklaus, M., Dubois, P., Shea, H., "Metal Ion Implantation for the Fabrication of Stretchable Electrodes on Elastomers", Advanced Functional Materials, Vol. 19, Issue 3, 470 - 478 (2009).

[8] M. Niklaus, M., Shea, H., "Electrical conductivity and Young's modulus of flexible nanocomposites made by metal ion implantation of Polydimethylsiloxane: the relationship between nanostructure and macroscopic properties", Acta Materialia, vol. 59, pp .830-840 (2011).

[9] Ohring, M., [Materials Science of Thin Films], Academic Press, (2002).

[10] Akbari, S., Niklaus, M., Shea, H., "Arrays of EAP micro-actuators for single-cell stretching applications", Proc. SPIE 7642, 76420H-9 (2010).

[11] Merlet, J.-P., [Parallel Robots, 2nd ed.], Springer, (2006).

[12] Eddings, M. A., Johnson, M. A., and Gale, B. K., "Determining the optimal PDMS-PDMS bonding technique for microfluidic devices," J Micromech. Microeng., vol. 18, 067001 (2008).

[13] Eddings, M. A., Gale, B. K., 2006 A PDMS-based gas permeation pump for on-chip fluid handling in microfluidic devices J. Micromech. Microeng. 16, 2396-402 (2006).

[14] Kofod, G., Paajanen, M. and Bauer, S. "New design concept for dielectric elastomer actuators", Proc. SPIE, 6168, 689-697 (2006).

[15] Niklaus, M., Rosset, S., Dadras, M., Dubois, P., Shea, H. R., "Microstructure of $5 \mathrm{keV}$ gold-implanted polydimethylsiloxane", Scripta Materialia, 59, 893-896 (2008). 